Revista

\section{Ciencia Económica}

Órgano de difusión del Seminario Permanente de la Academia de Teoría Económica

UNAM

José Narro Robles

Rector

Eduardo Bárzana García

Secretario General

Leopoldo Silva Gutiérrez

Secretario Administrativo

Javier de la Fuente Hernández

Secretaria de Desarrollo Institucional

Ramiro Jesús Sandoval

Secretario de Servicios

a la Comunidad Universitaria

Luis Raúl González Pérez Abogado General
1 de agosto de 2013

año $2 \bullet$ no. 3
Facultad de Economía

\section{Reflexiones sobre la Docencia}

Andjel, E., Caballero, E. ${ }^{\dagger}$, Ibarra, J. y Sandoval, J., 2013. Contenido de los cursos obligatorios de Macroeconomía: una propuesta. Ciencia Económica, 2(3), pp. 71-80.
Andrés Blancas Neria (Instituto de Investigaciones Económicas, UNAM) Jorge Carreto Sanguinés (Facultad de Economía, UNAM) Irma Escarcega Aguirre (Facultad de Economía, UNAM)

Carlos Guerrero de Lizardi (Tecnológico de Monterrey, Campus Cd. México) Rogelio Huerta Quintanilla (Facultad de Economía, UNAM) Carlos Ibarra Niño (Universidad de las Américas, Puebla) Javier Martínez Peinado (Universidad de Barcelona)

Carlos Maya Ambía (Universidad de Guadalajara) Comité Editorial
Revista Ciencia Económica • Publicación semestral http://www.economia.unam.mx/cienciaeco/ 


\section{Contenido de los cursos obligatorios de Macroeconomía: una propuesta}

\section{Eloísa Andjel Emilio Caballero ${ }^{\dagger}$ Jorge Ibarra José Sandoval}

Facultad de Economía, Universidad Nacional Autónoma de México (UNAM) <eandjel@unam.mx>,<ibacons@unam.mx>y<mansanjo@gmail.com>, respectivamente

doi: 10.22201/fe.24484962e.2013.v2n3.a5

\section{ANTECEDENTES}

\section{I}

T a teoría macroeconómica predominante (la más difundida), de fun$\amalg$ damentación neoclásica, evolucionó durante las últimas décadas reincorporando la concepción de un sistema de libre mercado, donde los protagonistas son agentes individuales que actúan con racionalidad plena, en un contexto que permite que así sea. En este sistema, el comportamiento racional de los individuos crea el escenario propicio para arribar a la asignación eficiente de los recursos productivos, así como a su utilización plena, teniendo esta última como límite a la libre voluntad de los participantes (consumidores-portadores de factores productivos), al tomar decisiones frente a las señales que genera el mercado, que es impersonal.

Para que la asignación eficiente se logre (y con ello la ocupación plena), el sistema de mercado debe operar sin ataduras, ni interferencias. Por tal razón, la participación del Estado en actividades productivas, regulatorias o con intenciones redistributivas es asumida, en principio, como algo inconveniente, por considerársele en la mayoría de los casos como una fuente de distorsiones y, finalmente, de resultados inferiores a los óptimos.

En el ámbito macroeconómico, el enfoque neoclásico presenta variantes. Se conforma de corrientes que recuperan la noción prekeynesiana de una economía que, en principio, contiene mecanismos que tienden a ubicar su funcionamiento en un nivel de plena ocupación de la capacidad productiva. Mecanismos que en un caso (nuevos clásicos) operan desde el corto plazo y en el otro (nuevos keynesianos) marcan sólo una tendencia de largo plazo sobre la cual la economía gravita. Entonces, los nuevos clásicos y nuevos keynesianos difieren entre sí, en torno a la fluidez de la 
tendencia de la economía a lograr y, en su caso, restaurar la plena ocupación, así como sobre las posibilidades de intervenir desde el Estado con este fin. Sin embargo, ambos enfoques comparten una fundamentación común que las acerca: la microeconomía neoclásica del agente racional, que viendo hacia el futuro y utilizando toda la información a su alcance, forja expectativas que, añadidas a las que forjan los demás sujetos que actúan de la misma manera, resultan ser en promedio acertadas, es decir, compatibles con la ocupación plena.

En este marco, se asume que las acciones de política de impulso al producto y al empleo estimulando la demanda agregada, o no son eficaces, en el caso más radical, o si acaso, según su versión menos estricta, se obtienen resultados positivos cuando los precios y los salarios no se muestran flexibles. Más, en el largo plazo, los precios y los salarios se ajustan y el producto tiende a restaurarse en su nivel "natural" de ocupación plena, por lo que la mirada de la política macroeconómica en el corto-mediano plazo no se fija tanto en mantener el pleno empleo de manera sostenida y puntual, sino en evitar la inflación.

Esta orientación del análisis convencional en realidad ha sido la reedición de una forma de pensamiento económico previamente existente y de larga tradición, que se remonta a los siglos XVIII y XIX, impulsado en las últimas décadas por diferentes actores sociales, con una energía renovada y contando con un marco doctrinario que ha sido eficaz. Y la renovación del pensamiento económico predominante ha sido desarrollada en su parte teórico-analítica bajo esquemas de gran complejidad formal. Esta posición se ha extendido a lo largo de ámbitos académicos de diferente renombre, en los que, por otra parte, ha tenido lugar una creciente exclusión de otras formas alternativas de teorización.

Desde luego que la economía neoclásica ha sido la dominante en los centros de enseñanza durante mucho tiempo. Primero la versión de síntesis neoclásica de Keynes y después las corrientes de sesgo prekeynesiano que prevalecen hoy en día. Lo diferente de las últimas décadas a sido la cerrazón de los economistas ortodoxos frente a las críticas y propuestas alternativas de teoría y política emanadas de corrientes que hoy se han dado en llamar heterodoxas, que son corrientes de largo aliento emanadas de las obras originales de los economistas clásicos, de Marx, Sraffa, Keynes, Kalecki, los institucionalistas y otros, así como de las elaboraciones posteriores de sus seguidores. Es decir, que en otros tiempos, digamos entre los años cincuenta y los setenta, aun con el predominio de la teoría convencional había más espacios de encuentro y debate entre economistas neoclásicos y no neoclásicos, en las universidades, en las 
revistas científicas, etcétera, y los estudiantes podían percatarse de las diferencias y darle seguimiento a las controversias, para, finalmente, guiarse por las explicaciones que les resultaran más convincentes.

En general, las posiciones heterodoxas, construidas bajo supuestos y posturas metodológicas diferentes, descansan en la presunción de que el sistema de mercado presenta fallas e insuficiencias de distinto tipo y que, en consecuencia, debería ser intervenido y regulado desde la sociedad misma a través del Estado. Esta orientación de la teoría, a partir de su posición crítica frente a la economía de libre mercado, y su contraposición con la teoría convencional ha dado pie a contribuciones y polémicas diversas que conforman un campo fértil y plural de análisis y de prescripción de políticas alternativas.

Algunas de las preocupaciones fundamentales de las orientaciones heterodoxas han sido situaciones adversas recurrentes, tales como la desocupación de los recursos productivos, la inestabilidad cíclica en los niveles de actividad -el estancamiento económico en muchos países- y la distribución concentradora del ingreso y la riqueza, fenómenos considerados como inherentes al funcionamiento de la economía de mercado y de los cuales la ciencia económica se debería ocupar, comprendiéndolos correctamente y derivando frente a ellos prescripciones adecuadas de política.

Las líneas de pensamiento heterodoxas se han seguido desarrollando, como siempre, en el seno de corrientes alternativas diversas de larga tradición, pero ante el cierre de filas de los impulsores de la teoría económica convencional han ocupado un segundo plano, más restringido, en los centros de investigación y docencia económicas, y durante mucho tiempo han visto reducir la influencia que llegaron a alcanzar en algún momento en la discusión pública y en la elaboración de programas y políticas.

II

El curso que ha seguido la economía de las naciones durante las últimas décadas ha entrañado la reestructuración institucional y la aplicación de políticas diseñadas en concordancia con lo establecido bajo el marco doctrinario y analítico neoclásico contemporáneo. Los resultados de todo este largo periodo han sido evaluados de diferente manera por economistas de distintas persuasiones.

Antes de que estallara la crisis económica actual, muchos economistas de la corriente predominante resaltaron frecuentemente y con insistencia el logro, durante digamos los veinte años previos, de una mayor estabilidad en el desempeño de las economías. En ello, se nos decía, fue 
esencial el avance realizado por la ciencia económica (entendida como la economía neoclásica), al proponer explicaciones del funcionamiento de la economía que dan sustento a las políticas aplicadas.

En cambio, desde las posiciones heterodoxas se venía señalando un crecimiento relativamente más lento y niveles de desocupación más elevados por los cuales, con diferencias entre países, se estaba transitando, así como la notable reconcentración que se producía en la distribución del ingreso y la riqueza, aunada al estancamiento o, en su caso, al franco retroceso de las retribuciones al trabajo. No menos importante fue, además, la alerta sobre los peligros del entramado financiero que se venía configurando.

La crisis económica que formalmente se reconoce a partir de finales del 2007 ha revelado muchas de las inconsistencias y los serios problemas incubados a lo largo del curso que siguieron las economías en las últimas décadas, y que fueron señaladas con anticipación en diferentes frentes de pensamiento crítico. Lo notable dentro de lo que ha ocurrido es que el pensamiento económico convencional no dio cabida o no prestó atención suficiente en sus elaboraciones teóricas ni en su agenda de investigación a la conformación de estos problemas que, al acumularse, desembocaron precisamente en la grave situación de los últimos años.

Las políticas aplicadas frente a este escenario de crisis han sido sumamente duras para los sectores más amplios de la población y, en ciertas vertientes, han entrañado el reforzamiento de las pautas de la redistribución inequitativa del ingreso. En medio de una situación que no apunta a corregirse, el panorama frente al futuro que nos ofrecen distintas predicciones no es de ninguna manera positivo. Lo paradójico es que, con algunas excepciones que no han marcado la pauta a seguir, estas políticas se han mantenido en la línea del pensamiento económico convencional prevaleciente.

En la situación actual, en el ámbito de la teoría económica resurge la importancia de la concurrencia de enfoques alternativos que compiten por explicar la realidad presente, cómo fue que se desembocó en el estado actual de cosas y qué programas y políticas deberían ser los más adecuados para enderezar el rumbo económico. En particular, la crítica severa hacia la teoría convencional ha aflorado en diferentes frentes, no solamente el académico; y dentro del académico no únicamente desde las posiciones heterodoxas, sino también de parte de destacados economistas surgidos de la corriente predominante misma. La ciencia económica, representada por el pensamiento predominante, ha perdido credibilidad, y esto es algo que no se puede negar. Vivimos un momento 
en el que se precisa volver a poner atención y darle la importancia debida a lo que nos ofrecen las explicaciones de los economistas que de distinta manera y en diferente grado disienten de la ortodoxia neoclásica, y no simplemente atender a las respuestas de los propios economistas convencionales.

En este contexto surge una inquietud sobre cómo se debe armar un programa de enseñanza de Teoría Económica. Entre quienes la impartimos en la Facultad de Economía de la UNAM existe una fuerte corriente de opinión que favorece la preservación de programas de estudio que ofrezcan un contenido plural, en contra de la idea de centrar los cursos de manera fundamental en la agenda de estudio neoclásica, tal y como ocurre en muchas otras universidades. Esta posición se deriva del propósito ampliamente aceptado en la Facultad de que, con la participación compartida con las demás áreas del plan de estudios, los estudiantes conozcan a lo largo de la carrera la diversidad del pensamiento económico actual.

Por lo que toca a los cursos obligatorios de Teoría Macroeconómica, estimamos que su contribución a la enseñanza plural estriba en ofrecer a los estudiantes los grandes temas del estudio del funcionamiento global de la economía presentados al menos bajo dos perspectivas: el enfoque convencional predominante, representado por los consensos y las diferencias de las posiciones nuevo clásica y nuevo keynesiana, y la perspectiva de ascendencia keynesiano-kaleckiana, representada por la corriente hoy llamada poskeynesiana.

Sobre esa base, una vez cubiertos los cursos obligatorios se propone abrir en el área terminal materias optativas en las que se pueda continuar el estudio de la macroeconomía, a un nivel más avanzado, ofreciendo, además, la posibilidad de concentrarse en la perspectiva analítica que a cada estudiante le resulte más interesante, entre las ofrecidas por profesores que participan de enfoques diferentes.

\section{COMPROMISOS CON EL CONTENIDO Y GRADOS DE LIBERTAD}

Con respecto a la propuesta que se presenta a continuación para los cursos de Teoría Macroeconómica I y II se entendería que cada profesor se compromete a tratar como mínimo los grandes temas enunciados, inicialmente denominados de manera genérica en términos de los aspectos y problemas fundamentales que aborda el análisis macroeconómico. Estos temas "gruesos" y su tratamiento según cada corriente definirían el contenido del "programa oficial" de la materia. Cada profesor podría 
diseñar, de la forma que le parezca más conveniente tanto el orden del desarrollo de estos temas como, en especial, la secuencia para considerar y comparar a las distintas corrientes que se proponen. De la misma manera, cada profesor decidiría sobre la bibliografía a utilizar. En lo que sigue se hace un enunciado de los grandes temas, aclarando que el punteo que sigue a cada uno de ellos no pretende ser exhaustivo.

\section{Teoría Macroeconómica I}

El curso de Teoría Macroeconómica I comenzaría explicando la naturaleza del enfoque macroeconómico, sus rasgos distintivos y sus limitaciones. También nos presentaría los fenómenos que son su objeto de estudio y el marco conceptual y contable dentro del cual se realiza el análisis. De manera destacada, también nos deberá informar sobre la existencia de los distintos enfoques de la teoría macroeconómica contenidos en los programas, partiendo de sus preconcepciones y supuestos fundamentales, así como de un esbozo de su estructura analítica diferenciada.

En su parte teórica, el curso estaría centrado en la explicación de la determinación del producto, el empleo y el nivel general de precios, en un contexto de corto-mediano plazo, en esquemas o modelos alternativos que reúnen a los factores de oferta con los de la demanda de diferente manera. Para ello, se revisarían perspectivas diversas que se pueden separar entre aquellas en las que predominan los factores de oferta y aquellas en las que predomina el lado de la demanda. Casi todo el análisis se desarrollaría en este momento en el marco de una economía cerrada, pero se pueden adelantar algunos aspectos elementales de la economía abierta.

En todos los casos, después de plantear los escenarios más sencillos y elementales, se analizarían las repercusiones macroeconómicas del gasto público y la tributación.

De la misma manera, la presencia y el papel del dinero y el financiamiento, distinto bajo las diferentes perspectivas, sería un tema a destacar en todo el análisis.

Una vez desarrollados los elementos de análisis sobre los temas anteriores, en el contexto de los modelos considerados se analizaría la contraposición entre los problemas del empleo y la inestabilidad de precios.

Finalmente, lo desarrollado hasta este punto permitiría considerar algunas explicaciones sobre la naturaleza, los objetivos y los instrumentos de la política macroeconómica que inciden sobre la producción total, el empleo y el comportamiento del nivel de precios. 


\section{TEORÍA MACROECÓNOMICA I}

TEMARIO

I. La naturaleza del enfoque macroeconómico y su objeto de estudio

- La perspectiva macroeconómica y la construcción de agregados

- Un avance de los grandes temas del análisis macroeconómico

- La construcción de modelos y la naturaleza de sus explicaciones

- Teoría y prescripciones de política

- Enfoques alternativos de la teoría macroeconómica

II. Un marco conceptual y de relaciones contables

- Descripción del funcionamiento global de la economía: los sectores institucionales y la generación de los flujos de producto ingreso y sus componentes

- Un marco contable para el análisis macroeconómico

- El problema de la medición de las variables macroeconómicas

- Conceptos relativos al empleo

- Limitaciones del marco conceptual para registrar la actividad económica

- Desempeño macroeconómico y bienestar

III. Demanda agregada y producto total y empleo

- Capacidad productiva y producción efectivamente realizada

- Producción mercantil y realización de la producción: el problema de la demanda agregada

- Producto total, empleo y desempleo

- La problemática del desempleo y la desocupación de capacidad instalada

- Expectativas y decisiones de gasto

- Decisiones de gasto privado (consumo e inversión) y demanda agregada

- El efecto multiplicador

- Gasto público, tributación y demanda agregada

- La política fiscal macroeconómica

IV. Dinero, financiamiento y demanda agregada

- El dinero y sus funciones en una economía capitalista

- El banco central, los bancos y la oferta monetaria

- Los mercados financieros

- Demanda de dinero y crédito

- Tasas de interés

- La demanda agregada y el producto con la presencia del dinero y el financiamiento

- La política monetaria y la demanda agregada

V. Oferta agregada y nivel general de precios

- Producción total y rendimiento de los factores

- Empleo y salarios 
- Empresas, estructuras de mercado, márgenes de ganancia y nivel general de precios

- Márgenes de ganancia, distribución del ingreso y salarios reales

- Esquemas alternativos que relacionan a la producción total con el comportamiento del nivel general de precios: curvas de oferta agregada

VI. Modelos completos de oferta y demanda agregadas: producto, empleo y nivel general de precios

- Un modelo nuevo-clásico

- Un modelo de la nueva síntesis keynesiana neoclásica

- Un modelo poskeynesiano

- La inflación y sus consecuencias

- Inflación y desempleo bajo los diferentes modelos

- Políticas antiinflacionarias del lado de la oferta y del lado de la demanda

\section{Teoría Macroeconómica II}

El curso de Teoría Macroeconómica II retomaría la explicación del producto total, el empleo y el nivel general de precios, pero en el contexto de una economía abierta al exterior, por lo que ahora las relaciones económicas con el exterior aparecerían como un condicionante más de la producción y el empleo totales, y también como elementos que se interrelacionan con el nivel general de precios.

El análisis de la economía abierta muestra diferentes resultados en función de los supuestos que se hagan sobre el grado de competencia al interior de la economía, así como el régimen cambiario considerado y el grado de movilidad de capital entre países. Aunque se considerarían distintas variantes con respecto a estos casos, el énfasis se pondría en el caso de una economía con competencia imperfecta a su interior y un banco central que en principio fija la tasa de interés, analizando los casos que se presentan con ambos regímenes cambiarios, fijo y flotante, y con movilidad de capital perfecta e imperfecta, juzgando la pertinencia de cada uno para países y momentos particulares.

También se retomaría el tema de la inflación, pero ahora en el contexto más complejo de la economía abierta, en el que se añade su interacción con el tipo de cambio y el balance externo.

Finalmente, se revisarían las prescripciones de política económica en un escenario en el que concurren las preocupaciones por el empleo, la estabilidad de precios y un estado manejable de los pagos al exterior. 


\section{TEORÍA MACROECONÓMICA II \\ TEMARIO}

I. Las relaciones económicas con el exterior, su tipología y la problemática de los pagos externo

- Tipología de transacciones y pagos con el exterior: comercio, pagos factoriales, transferencias y operaciones con activos físicos y financieros

- La balanza de pagos y la interpretación de sus resultados

- La viabilidad de la situación de pagos frente al exterior

II. El marco contable para el análisis de la economía abierta: balanza de pagos y flujo de producto-ingreso

- Flujo de producto-ingreso y balanza comercial

- Producto interno e ingreso nacional

- La relación ahorro-inversión entre el sector privado, el gobierno y el resto del mundo

III. El mercado de cambios, el tipo de cambio y la competitividad en el comercio mundial

- El concepto de tipo de cambio nominal y sus derivaciones

- Oferta y demanda de divisas: sus determinantes

- Teorías de la determinación del tipo de cambio

- Los regímenes cambiarios y sus implicaciones

- El tipo de cambio real y la competitividad externa en materia de precios

IV. Demanda agregada, determinación del producto y balance externo en cuenta corriente en una economía abierta con precios dados: la restricción externa

- Demanda agregada, pagos al (y desde el) exterior y demanda de bienes producidos dentro del país

- Determinación del producto que encuentra salida en el mercado en la economía abierta

- Incompatibilidad entre la situación de pagos al exterior y la situación interna de producción total y empleo

- Recuperación de una situación viable de pagos frente al exterior: las políticas de contracción del gasto

- Recuperación de una situación viable de pagos frente al exterior: la política cambiaria

V. Los movimientos internacionales de capital y sus determinantes

- Rentabilidad de las inversiones productivas

- Diferenciales de tasas de interés

- Expectativas sobre el tipo de cambio

- Incertidumbre y confianza en las inversiones en el exterior 
VI. Balance externo, movimientos de capital y mercado monetario

- Manejo monetario interno y movimientos de capital

- Opciones y restricciones a la política monetaria

VII. Determinación del producto y la balanza de pagos completa bajo diferentes condiciones de régimen cambiario y de movilidad de capitales

- Movimientos de capital, tipo de cambio, competitividad externa y producto total

- Equilibrio interno y equilibrio externo desde la óptica de la demanda total y la determinación del producto

VIII. El lado de la oferta y el nivel general de precios en la economía abierta

- Comercio internacional, insumos importados y nivel de precios

- Tipo de cambio real y distribución del ingreso interno

- Margen de ganancia, tipo de cambio real y salario real

IX. Inflación y desempleo en la economía abierta, problemática y políticas

- Negociación salarial, salario real y competitividad externa

- Balance externo y desbalance distributivo

- Política cambiaria e inflación interna

- Políticas en torno a una triple problemática: desempleo, inflación y desequilibrio externo 